\title{
Alegorías de la Nación
}

\section{y Cuerpos Femeninos. \\ Una Discusión sobre la}

\section{Construcción de la Historia}

\section{en Cambio de armas, de}

\section{Luisa Valenzuela}

Rauf Neme Sánchez ${ }^{*}$

Universidad Católica Sedes Sapientiae

rneme@ucss.edu.pe

Fecha de recepción: agosto de 2015

Fecha de aceptación: octubre de 2015

Resumen: La premisa de Fredric Jameson, “iHistoricemos siempre!”, en The Political Unconscious. Narrative as a Socially Simbolic Act, será el punto de partida para el análisis del libro de relatos Cambio de armas de la escritora argentina Luisa Valenzuela. Según Jameson, todo texto es político en el sentido de que busca resolver en el espacio de la ficción una contradicción existente en la realidad social y, por ende, su deseo es intervenir en la Historia. La intención del presente estudio es justamente examinar el subtexto que se deriva de la figura del cuerpo femenino como espacio textual para construir una lectura alegórica de la nación y demostrar cómo la construcción de esta alegoría permite cuestionar el discurso oficial sobre la Historia que surge desde los espacios de poder.

* Rauf Neme Sánchez estudió Literatura en la Pontificia Universidad Católica del Perú. Es miembro asociado de la Asociación Peruana de Literatura Comparada (ASPLIC) y del International Comparative Literature Association (ICLA). Ha sido miembro del comité organizador y participante en los coloquios internacionales de Literatura Comparada organizados por el ASPLIC en los años 2004, 2005, 2008 y 2010. También participó como ponente en el 19 Deutscher Hispanistentag en la Universität Münster, Alemania, en el año 2013. En otras actividades, ha musicalizado el cortometraje-documental The Calm de Fernando Vílchez, selección oficial en el festival de cine de Berlín a mejor cortometraje y ganador del XV Festival de Cine de Lima en la categoría de mejor corto-documental. 
Palabras clave: Poder, alegoría y nación, literatura argentina contemporánea, cuerpo y violencia, dictadura, Historia, realidad social.

\title{
National Allegories and Female Bodies. A Discussion on the Construction of History in Cambio de armas, by Luisa Valenzuela.
}

\begin{abstract}
The premise of Fredric Jameson, "Always historicize?", in The Political Unconscious. Narrative as a Socially Simbolic Act, is our starting point for the analysis of the story book Cambio de Armas of the Argentinian writer Luisa Valenzuela. According to Jameson, every text is political in the sense that it seeks to address in the space of fiction an existing contradiction in the social reality and, therefore, its desire is to interfere in History. Our intention is precisely to examine the subtext that is derived from the figure of the female body as textual space to build an allegorical reading of the nation and demonstrate how the construction of this allegory challenges the official discourse on History which emerges from the spaces of power.
\end{abstract}

Keywords: Power, Allegory and Nation, Argentinian Contemporary Literature, Body and Violence, Dictatorship, History, Social Reality.

\section{Introducción}

ambio de armas es un libro que se contextualiza en la conflictiva y represiva experiencia
política de la Guerra Sucia en Argentina (1976-1982). Este periodo traumático de la historia del país del sur se caracterizó porque el régimen militar afectó el orden institucional del país y alteró todas las esferas de la vida cotidiana de los argentinos. Los atentados contra los opositores se volvieron una constante y la represión de las voces disidentes se extendieron a escritores e intelectuales que eran identificados como enemigos por el gobierno militar.

En este confuso y beligerante panorama, los relatos de Cambio de armas (1982), de Luisa Valenzuela, manifiestan mediante los recursos de la ficción la violencia de la dictadura en el cuerpo femenino torturado y la construcción de un poderoso andamiaje represivo en el terreno del lenguaje. Esta lectura incide en mostrar cómo esta construcción alegórica en torno al cuerpo intenta abordar las contradicciones presentes de la realidad social y proyectan una acción de empoderamiento para intervenir en la Historia y dar un revés al discurso de poder que oprime a la sociedad. 


\section{Alegoría e Interpretación}

$\mathrm{E}$ n este análisis se seguirán las consideraciones que propone Fredric Jameson en The Political Unconscious (1994). Según este autor, el horizonte absoluto de cualquier lectura o interpretación de los textos literarios es la interpretación política. En ese sentido, esta interpretación no es un método auxiliar ni suplementario a otras formas de acercarse al texto.

Para Jameson, los textos literarios pueden articular trasfondos políticos, pero siempre entendidos desde la concepción de la Historia como un solo "relato ininterrumpido". Así, la interpretación del texto puede permitir vislumbrar ese único trasfondo: "la realidad reprimida y enterrada de esa historia fundamental" (Jameson, 1989, p. 15), lo que se identifica con el inconsciente político. Este, además, permite comprender los "artefactos culturales como actos socialmente simbólicos" (Jameson, 1989, p. 18).

Es en esta formulación que la alegoría adquiere un valor significativo, pues es el modelo sobre el cual los textos se construyen y pueden ser leídos "como tipificaciones de elementos en otros niveles" (Jameson, 1989, p. 20) y por lo que "los significados de los relatos alegóricos son una dimensión persistente de los textos literarios y culturales precisamente porque reflejan una dimensión fundamental de nuestro pensamiento colectivo y de nuestras fantasías colectivas sobre la historia y la realidad" (Jameson, 1989, p. 29). Asimismo, se debe indicar que la alegoría es parte de un sistema de cuatro niveles: (a) anagógico, que supone una lectura política y puede dar cuenta del significado colectivo de la historia; (b) moral, que es la lectura psicológica desde la perspectiva del sujeto como individuo; (c) la alegoría, en donde se propone el código interpretativo; y (d) el nivel literal que es el referente histórico textual (Jameson, 1989).

Así, para Jameson, un asunto fundamental dentro del proceso de interpretación, a diferencia del método que deriva de las ciencias sociales, es establecer que los textos como actos simbólicos pretenden ofrecer "soluciones a determinadas contradicciones" de lo que está inscrito como Real. El autor sostiene:

Hemos supuesto que, para ser consecuentes, la necesidad de la voluntad de leer los textos literarios o culturales como actos simbólicos debe aprehenderlos necesariamente como soluciones a determinadas contradicciones (...) La 
exigencia metodológica de articular la contradicción fundamental de un texto puede verse pues como una prueba de lo completo del análisis: por eso, por ejemplo, la sociología convencional de la literatura o de la cultura, que se limita modestamente a la identificación de motivos o valores de clase en un texto dado, y siente que su trabajo ha quedado hecho cuando muestra que un artefacto dado «refleja» su trasfondo social, es profundamente inaceptable. (Jameson, 1989, p. 65)

En ese sentido, la interpretación es una reescritura del texto literario al mismo tiempo que es un reestructuración de un "previo subtexto histórico o ideológico" (Jameson, 1989, p. 66). Este no puede entenderse como algo exterior al texto, pues lo Real está vinculado al lenguaje de un modo intrínseco. La Historia, en esa línea, solo puede ser aprehendida luego de ser textualizada y narrativizada.

En esta línea, la propuesta de Jameson permite abordar la narrativa de Valenzuela como un artefacto cultural y simbólico que propone en el terreno de la ficción una solución temporal al complejo problema social derivado de un proceso autoritario y opresivo. En este caso, corresponde relacionarlo con el contexto de la dictadura argentina. Así la ficción busca una intervención en la Historia, en la región de lo Real.

\section{Cuerpo y Escritura}

\footnotetext{
C uál es la importancia en relacionar el cuerpo con el poder? ¿Cómo el discurso ficcional construye una relación de significado entre cuerpo y poder? Finalmente, ¿Cómo la ficción ocuparía ese rol crítico y cuestionador frente al poder? La relación simbólica entre cuerpo y poder es un lugar común dentro de la historia del pensamiento occidental. Se puede afirmar que ha funcionado como mecanismo para crear mitos, para sustentar un culto político (Rader, 2006), para abordar la idea misma de las estructuras del poder como un cuerpo social y administrativo (Kantorowicz, 2012); es por ello que esta relación metafórica entre cuerpo y poder ha recibido una atención especial siempre desde la filosofía política para explicar cómo surgen y funcionan las propias concepciones sobre el orden político.
}

En la literatura, esta metáfora es un tema frecuente debido a las múltiples posibilidades creativas y especulativas que ofrece como objeto de ficción. Para precisar en 
el caso de la literatura hispanoamericana, según Olivia Vásquez-Medina (2013), hay una larga tradición sobre la escritura del cuerpo: "la novelística de mediados del siglo XX lo habría consolidado ya como topos para reflexionar sobre una problemática específica: la nación” (p.16). Dicha reflexión, desde la novela histórica, por ejemplo, pone en relieve que el empleo del cuerpo articula una crítica bifronte, pues actúa sobre la ficcionalización del pasado y también "desde el presente donde se escribe" (p.17).

De ese modo, Cambio de armas es un libro que utiliza alegóricamente la figura del cuerpo femenino como el espacio en donde se va a textualizar la violencia del poder androcéntrico que representa la dictadura. En ese sentido es un libro disidente, crítico, inscrito dentro de una tradición de textos que buscan intervenir en la Historia. Igualmente, intenta dejar constancia en los espacios de la ficción de las serias contradicciones de la realidad social, política y moral de su tiempo.

Luisa Valenzuela escribe sobre el cuerpo y "escribe con el cuerpo" de una manera consciente. ${ }^{1} \mathrm{Su}$ narrativa es política e incide en proyectarse desde las esferas íntimas o privadas de los sujetos, como son las relaciones maritales, aventuras eróticas o uniones fallidas, cuyo subtexto ideológico se manifiesta en actos de "empoderamiento":

The politics of the private sphere as well as that of the public is a motif which permeates her work, focusing as it does on the relationship of the opresor and the opressed, those who govern and those who are governed, the social and the psychological, male and female. Here is a narrative in which writing is an instigator and 'disturber of ideas', an act of personal empowerment which gives rise to political consciousness. (Saltz, 1987, p.61)

Asimismo, según Muñoz (1992), el conocimiento del cuerpo permite entender la postura de Valenzuela frente a la escritura, en el sentido de que construye un lenguaje femenino desde la exploración de las "energías de la mujer, su poder y potencia localizadas en las regiones de su femineidad, cuya representación será codificada en un cuerpo textual femenino" (p. 63). El cuerpo textual femenino no es otra cosa que el proceso de la escritura como "la autoconciencia

1 En palabras de la escritora, escribir implica una actitud vital: "Es más bien un trabajo conjunto de la cabeza y lo otro, aquello que nos impulsa a movernos, a bailar, a respirar y exhalar, es decir por lo tanto acarrea a la cabeza y con ella al raciocinio a explorar caminos impensados que suelen asustarnos" (Hartry, 2010, p. 339). 
que resulta del conocimiento de su propio cuerpo" (1992, p. 63). Cabe agregar que es ese el modo en que lo conceptualiza el ginocentrismo de orientación francesa.

Así, en esta necesidad de problematizar la representación de la mujer y también su relación con la escritura, Cambio de armas ofrece una solución temporal para resolver las contradicciones del contexto: apropiarse del lenguaje para luego resignificarlo desde las coordenadas propuestas que surgen del conocimiento del cuerpo femenino. Es mediante esta labor de resignificación que el cuerpo femenino buscará liberarse de las fuerzas opresoras. Esta será su acción de empoderamiento y su intervención en lo Real.

\section{Cuarta Versión, el Problema de Escribir la Historia}

$\mathrm{E}$ n el conjunto de relatos de Cambio de armas es posible verificar la necesidad de reescribir la Historia, pero desde un discurso que articula elementos subversivos entendidos aquí como fuerzas liberadoras. La construcción de este discurso entra en permanente conflicto con la "versión" oficial que se identifica con el poder de la dictadura y su lenguaje androcéntrico. Para analizar estos aspectos, se tomará como referencia dos cuentos que, para el presente estudio, son significativos: "Cuarta versión” y "Cambio de armas".

"Cuarta versión" abre el conjunto de cuentos y, en la perspectiva de este estudio, alude desde su título el ejercicio de la constante reescritura, cuestión que se reafirmará en las intervenciones de la narradora-autora. Su argumento se centra en la aventura extramarital de Bella y Pedro: ella es una actriz cuya superficialidad y belleza atraen a Pedro, un diplomático de modales atildados y casado. La historia de ambos transcurre por lo general en el interior de la embajada, en donde Pedro siempre ofrece cocteles y recepciones, a las cuales asisten personajes importantes como también artistas para socializar. Sin embargo, en los exteriores de este espacio, el ambiente es distinto: existe un miedo constante, la represión por parte de los militares y las permanentes persecuciones es una información que dosificadamente va aflorando en las conversaciones de los asistentes a las reuniones en la embajada.

En su construcción, "Cuarta versión” permite identificar una doble línea argumental. La primera se centra en el problema de la escritura de la Historia. La otra se ocupa de la relación amorosa de Bella y Pedro, en donde se nota la función del cuerpo del protagonista que se convierte también en otro objeto escrito.

Sobre la primera línea argumental, se puede señalar que el relato inicia con un prólogo que expone una declaración de motivos. De esta manera, se manifiesta el conflicto en el cual 
se halla el narrador: textualizar la Historia, prodigarle un orden, una linealidad que le permita establecer una secuencia entre las distintas experiencias que configuran la historia personal de Bella, el personaje de la narración:

Leo y releo estas páginas sueltas y a veces el azar reconstruye el orden. Me topo con múltiples principios. Los estudio, descarto y recupero y trato de ubicarlos en el sitio adecuado en un furioso intento de armar el rompecabezas. De estampar en alguna parte la memoria congelada de los hechos para que esta cadena de acontecimientos no se olvide ni se repita. Quiero a toda costa reconstruir la historia (...). (Valenzuela, 2007, p. 12)

En este fragmento queda en evidencia un aspecto que Fredric Jameson (1989) ya había notado como relevante en la comprensión de la Historia, en el sentido de que "el problema de la representación, y muy particularmente el de la representación de la Historia: [es] un problema esencialmente narrativo, una cuestión de la adecuación de cualquier marco del relato en que puede representarse la Historia" (1989, p.41). Este relato no puede ser producto de una entidad completamente externa, solo se puede configurar como producto de la interpretación del narrador: "leo", "releo", "estudio", "descarto" y "recupero". Una interpretación que es el resultado de un esfuerzo dialéctico, entre distinguir las respectivas versiones para llegar a una conclusión de lo Real, y en donde siempre existe una tensión entre lo que está escrito y los silencios propios de lo "escamoteado" de los papeles de Bella:

Lo que más me preocupa de esta historia es aquello que se está escamoteando, lo que no logra ser narrado (...) Lo escamoteado no es el sexo, no es el deseo como suele ocurrir en otros casos (...) Los asilados políticos. De ellos se trata aunque estas páginas que ahora recorro y a veces reproduzco sólo lo mencionan de pasada, como al descuido. (Valenzuela, 2007, pp. 34-35)

Estos puntos que han sido silenciados adrede son los que configuran a un narrador preocupado en dilucidar e interpretar correctamente los hechos no verbalizados y, por ende, borrados de la historia de Bella. Es por ello que se atribuye el papel del autor: "No hay autor y ahora la autora soy yo, apropiándome de este material que genera la desesperación 
de la escritura” (Valenzuela, 2007, p.35). Esta autoría es necesaria para concluir el proyecto textualizado sobre aquella "realidad" en donde la interpretación aún no tiene un sentido fijo.

Esta es la ansiedad que agobia a la narradora, de quien ahora la marca de su género la individualiza. Por tal motivo, su intento de armar el rompecabezas origina en ella una reacción de furia frente a su impotencia "de la escritura”. Si anteriormente se señaló la función del ginocentrismo en la escritura de Valenzuela, aquí se ve que la "apropiación” del lenguaje predominantemente androcéntrico es limitante y no representa en sí la vía para poder representar la realidad desde el punto de vista femenino.

Por otro lado, estas distintas versiones, para Sharon Magnarelli, no son la principal preocupación a tener en cuenta sino las lecturas posibles que derivan de los distintos planos que articula. Estos son las que siguen: (a) la historia política que es un subtexto dentro de la historia de amor; (b) la construcción de una trama en donde el lector y el autor asumen el rol del detective para reconstruir la verdad de los acontecimientos; (c) el género del cuento de hadas; y (d) el acto de escribir (Sesana, 2003). De ese modo, se puede notar que esta diversidad de planos es producto también de que estructuralmente, como se señaló al inicio de esta sección, existe una división de espacios, interior (embajada) y exterior, que se oponen binariamente. Así, se puede decir que los campos de significado del interior se asocian con la libertad, la socialización, la diversión, el deseo (de Bella y Pedro), a diferencia del exterior que comprende la opresión, la persecución y la muerte.

El personaje de Bella transita entre ambos espacios, pero su presencia es contaminante en el espacio interior, pues tras de sus acciones subyacen las tensiones propias del exterior que van a desequilibrar el aparente orden que existe en la embajada. Es ahí donde la lectura política del cuento adquiere cierta dimensión, pues se observa a un personaje desdoblado: Bella, la actriz víctima del deseo por un hombre casado, y Bella, la activista política, o la subversiva si se ve desde el ángulo de los militares, que se preocupa en utilizar su vínculo con Pedro para conseguir asilo a los perseguidos políticos. De esta manera, el espacio de la embajada empieza a contaminarse del miedo y el tenebrismo político que existe en los exteriores.

Sin embargo, lo realmente importante frente a este material textual es la autoría que ha quedado inconclusa, por lo que el narrador busca legitimar su interpretación del material frente a las otras versiones que puedan derivarse, y en ese sentido fijar el sentido. Aquí subyace el subtexto ideológico que propone el primer cuento de Cambio de armas: las batallas por fijar el sentido de la Historia. Esta es una de las principales tensiones en 
el contexto político de una dictadura, pues sus mecanismos de propaganda intervienen y narran los hechos desde su punto de vista "oficial".

Frente a ello, la literatura se convierte en un discurso que desautoriza, contradice y cuestiona el poder desde los márgenes de la ficción. Es por ello que la narradora asume la responsabilidad de la autoría con la misma convicción política de Bella, su rol es también subversivo, y desde su lado, compromete al lector. Sobre este punto, Hartry (2010) propone que existe en la narración de Valenzuela un acto cooperativo con el lector, a manera de un "lector cómplice” en su reconstrucción de la narración. La investigadora afirma:

Este tipo de escritura experimental caracteriza a Valenzuela, que, además, usa la ruptura del lenguaje y de la forma de contar tradicionales para denunciar las convenciones, en este caso lingüísticas, llegando al punto de destruirlas. Esto, a su vez, lleva a la liberación de la represión mediante la libertad de expresión. (Hartry, 2010, p.337)

La anterior precisión clarifica más la visión sobre el trabajo de esta narradora. Querer superar las convenciones frente a las limitaciones que plantea el lenguaje es, desde el punto de vista del presente estudio, una toma de posición política frente a la subordinación que el discurso androcéntrico ha impuesto sobre la identidad femenina (discurso que, dicho sea de paso, emana de la dictadura que lo legitima).

La segunda línea argumental, la relación de Pedro y Bella, enfatiza la corporalidad de Bella, pues a partir de su cuerpo se puede lograr una resignificación del lenguaje que "desespera" a la narradora-autora. Del mismo modo, es también a partir de ella que se puede lograr esa síntesis dialéctica para poder cerrar el ciclo de los acontecimientos (hay que recordar que la narradora ha decidido apropiarse de su versión, reordenarla e interpretarla para intervenir en lo Real). En ese sentido, el cuerpo de Bella puede ser un criterio para dirigir la interpretación de la Realidad y la fijación del sentido en la escritura de la Historia:

Hay un punto en donde (...) una pasa a ser personaje de ficción o todo lo contrario, el personaje de ficción anida en nosotros y mucho de lo que expresamos o actuamos forma parte de la estructura narrativa, de un texto que vamos escribiendo con el cuerpo como una invitación. (Valenzuela, 2007, pp. 12-13, el subrayado es nuestro) 
Así, según la lógica del relato, la simetría entre la historia ficcional y la Historia está definida por una estructura narrativa que en esencia es textual, pero en donde también participa el cuerpo. Se podría señalar que estas alusiones intentan construir progresivamente en el conjunto de relatos la alegoría del cuerpo femenino como instrumento y luego como espacio de la escritura. Esto es, su causa eficiente, su primer punto de partida para el proceso de subversión y liberación de la escritura femenina.

Para que Bella pueda narrar mediante su cuerpo es importante el rol que se le adjudica dentro de la historia. A diferencia de los demás, en ella se destaca su naturaleza performativa por ser una actriz, de modo que podría decirse que "siempre interpreta el papel”.

(...) los rasgos de Bella se irán delineando con el correr de las admiraciones. De todos modos, ni tan Bella como indicaría su nombre ni tan ¿sofisticada? Aunque a veces algo de eso hay, sobre todo cuando juega su papel o simplemente cuando opta por mostrarse:

- Mi papel es estar viva. (Valenzuela, 2007, p.16)

Este "interpretar el papel” es significativo, pues se incluye dentro de un conjunto de estrategias que enfatizan la reconfiguración variable del personaje. De esa forma, es posible decir que la naturaleza performativa de Bella permite todo un ejercicio de resignificación constante derivado del hecho de que su cuerpo se convierte en signo, en un espacio que funciona como escenario teatral. Sobre este último punto, se puede anotar que el acto performativo de Bella también es subversivo porque busca enfrentarse a la censura de la dictadura mediante la puesta en escena y el enmascaramiento:

(...) el espectáculo que estaba a punto de estrenar y que llevaba por título El todo por el todo, representación unipersonal en dos actos y montones de actitudes, con todas las máscaras izadas como velas. Espectáculo concebido para invitar al público a jugarse tratando de burlar las barreras de censura mientras la posibilidad todavía existiera, apurándose antes de la represión (...). (Valenzuela, 2007, p. 49) 
Esta alusión a la puesta en escena dentro de la narración es un recurso metaficcional para revelar la verdad que se esconde en los entresijos de la ficción. En ese sentido, se trata de proyectar la misma condición que hay entre la puesta de escena de Bella y la narración de Cambio de armas. Es decir, ambos son textos políticos que recurren a la ficción para poder superar la censura e intervenir en la Historia.

Otro detalle que se agrega es su condición de apelar a las máscaras. Sobre ello, Bilbija (1996) en su análisis sobre "Ceremonias de rechazo", otro cuento incluido en Cambio de armas, señala el enmascaramiento mediante el maquillaje como una nueva semantización de la identidad. Esta estrategia, desde su lectura, es una forma de subversión psicopolítica frente al rol que el discurso androcéntrico de la dictadura militar le ha asignado a la mujer, tema que sin duda es central dentro de la constitución de los demás relatos: “(...) el texto es la máscara y su forma refleja la desintegración de la identidad unificada y estable” (p. 103).

En el caso de "Cuarta versión”, la máscara es el recurso para poder representar y en ese sentido redefinir su papel. Esto se da no solo en la puesta en escena, sino también en el orden exterior: actriz, amante, mujer superficial, activista política, perseguida política y víctima. Entonces, visto esto, se puede afirmar que el personaje de Bella al romper con su identidad está siempre en una actitud subversiva y transgresora en busca de su propia resignificación mediante el acto performativo o de enmascaramiento.

\section{Cambio de Armas, Lenguaje y Cuerpo Torturado}

l cuento "Cambio de armas" plantea como eje temático un cuestionamiento del lenguaje
a nivel del significado. Esta tensión a nivel del lenguaje es una dimensión más de los conflictos y cuestionamientos derivados sobre el poder que emana de la dictadura militar; en el sentido de que la dictadura legitima en el orden social la funcionalidad de este discurso que subordina a la mujer. Además, el relato problematiza asuntos como la identidad y la violencia en un entretejido de relaciones tortuosas que componen el conflicto del relato. En este texto, el cuerpo femenino se convierte en un medio para expresar las contradicciones del poder, en clara alusión también al "cambio de armas" que busca el sujeto femenino para liberarse de la opresión del discurso androcéntrico.

Sobre este "cambio de armas", Kaplan (2007) es enfática al señalar que el propósito que dirige la narrativa de Valenzuela es demostrar que "las mujeres sólo tendrán control sobre la palabra cuando 'entiendan' su propio cuerpo y que la escritura femenina debe ser consciente 
del cuerpo y de las fantasías de las mujeres. 'Escribir con el cuerpo' es la fórmula que propone" (p.67). Así, se podría derivar que el cambio de armas Real y su intervención en la Historia dependerá de cómo el sujeto femenino pueda apropiarse del lenguaje, resignificarlo, como se planteó en el análisis del primer cuento. Por ello, es posible afirmar, siguiendo la lectura de Kaplan, que el relato

no sólo problematiza la idea de un lenguaje con un sentido único sino que coloca al lenguaje en el lugar de la resistencia y de la apertura hacia una transformación social, en el supuesto caso de que la mujer logre sobrepasar la dificultad de acceder al simbólico reino del lenguaje sin simultáneamente ponerse bajo borradura, arriesgándose a ser malentendida o confirmando las representaciones patriarcales que preexisten en su habla. (Kaplan, 2007, p. 74)

El relato presenta como argumento la relación conyugal de Laura y Roque. Se sugiere que ella es víctima de su propio esposo, un coronel, quien la tortura en aquella casa en la que está cautiva. Laura, el personaje protagonista, aparece como una mujer amnésica y, por ende, su historia personal será paulatinamente revelada. Ella inicia su proceso de reconocimiento desde el punto de vista del otro, que es quien le atribuye su nombre: "Ella acepta las manos tendidas, inclina la cabeza ante el nombre de Laura también aceptándolo y él y sus colegas se sientan en los sillones y empiezan a examinarla” (Valenzuela, 2007, p. 127). Su verdadera identidad está borrosa y no aflorará hasta el final del relato para configurar también, en términos formales, el clímax en la resolución del conflicto.

Sobre esta amnesia, la crítica ha interpretado que puede identificarse como una alegoría colectiva (Hartry, 2010) sobre la falta de memoria en las naciones golpeadas por los regímenes autoritarios. $\mathrm{O}$ también sobre la ausencia adrede que existe en la construcción de la Historia desde el discurso oficial del poder. Sobre este último punto se puede seguir la idea de Callejo (1985), quien sostiene que en este relato es evidente que hay una alegoría política, de cómo el poder de la dictadura "hace perder la memoria a golpes [al pueblo], igual que a Laura [así el pueblo es] forzosamente amnésico, pero al que se le obliga a pensar en el dictador como padre de la patria, padre benefactor" (pp. 579-580).

En la misma línea, Jara (2007) propone que la falta de memoria es un elemento que Valenzuela asocia con la figura del "desaparecido". De esa forma, su fin es denunciar las prácticas regulares de la dictadura militar en Argentina en relación con la narración de la Historia: 
En "Cambio de armas" Valenzuela construye un yo condenado a la desaparición, a estallar en la figura de un otro indefinible, ajeno y siempre presente. Entonces, desaparecido el yo, queda abolida la memoria, borrándose, de este modo, todo vestigio de una historia individual o colectiva. (Jara, 2007, p. 223)

Por otro lado, para Kaplan (2007) esta amnesia tiene también un vínculo con el cuerpo femenino. En ese sentido se quiere describir la condición de la falta de memoria de Laura desde la relación entre lo textual y lo corpóreo: "El primer adjetivo que aparece, en la segunda línea, por un lado da la marca de género y por otro, corporiza esta desposesión: desnuda de recuerdos" (p. 113, el subrayado es nuestro).

Considerando lo anterior, Laura para afirmarse en el universo ficcional del relato debe empezar su proceso de adquisición del conocimiento. Por ello, se enfatiza cómo este proceso debe empezar por el lenguaje. Formalmente, se puede interpretar esto desde los subtítulos que plantea el cuento como experiencias de conocimiento a través de los siguientes términos: las palabras, el concepto, la fotografía, los nombres, la planta, los espejos, la ventana, los colegas, el pozo, el rebenque, la mirilla, las llaves, las voces...

Asimismo, las relaciones de designación se convierten en el único asidero para conservar la realidad y verdad de las cosas en este universo ficcional. Laura puede dar nombre a los objetos como si fueran operaciones sencillas que surgen sin necesidad de saber cómo operan en el fondo. Sin embargo, no puede designar la verdadera identidad del hombre que la posee:

Después está el hombre: ése, él, sinnombre al que le puede poner cualquier nombre que se le pase por la cabeza, total, todos son igualmente eficaces y el tipo, cuando anda por la casa le contesta aunque lo llame Hugo, Sebastián, Ignacio, Alfredo o lo que sea. (Valenzuela, 2007, pp. 113-114)

La acotación mediante la voz del narrador, "todos son igualmente eficaces", adquiere sentido y relieve cuando se sabe que Roque, el esposo torturador, es un coronel. En este pequeño intersticio la voz del narrador sugiere que en la dictadura se elimina la individualidad, pues esta se reduce a un espíritu de cuerpo, de grupo, de identidad uniforme y homogénea. Ahí radica su eficacia. Todos estos rasgos: perder la memoria, la ausencia de 
historia, la falta de un reconocimiento, pueden ser claves para la construcción de personajes que pretenden ser metáforas del desorden que vive una nación, aunque en el discurso oficial se señale que la dictadura se dio forzosamente como única solución para imponer el orden.

Ya antes se ha mencionado que la escritura femenina ha volcado sus estrategias para redefinir la identificación de la mujer desde su corporalidad. Laura se formula la pregunta sobre si su tiempo de florecimiento ha pasado o está próximo a llegar: “¿Cuándo habrá brillado el esplendor de ella [la planta]? ¿Habrá pasado ya el momento o estará por llegar?” (Valenzuela, 2007, p. 122). Se puede entender aquí también la situación del país, en el ideario común sobre si fue esplendoroso el pasado o de si es necesaria la tarea de reconstrucción que se proyecta hacia el futuro; pues el presente al igual que la planta es un estado marchito.

Lo mismo podría afirmarse en otro cuento, "Ceremonias de Rechazo", donde el propósito del personaje, Amanda, al sembrar en el jardín exterior que posee, muestra no solo la tarea de redefinir su identidad (el jardín interior se amalgama con el jardín exterior según la voz del narrador) sino también la necesidad de que su amante, Coyote, no intervenga más en él. Aquí no solo está presente la idea de un discurso alternativo femenino frente al hegemónico, sino también es una clara posición frente al poder. La reconstrucción nacional dependerá en gran parte de la ausencia del Coyote, que representa a la dictadura, y de la acción de empoderamiento de Amanda.

En cierto modo el texto se construye sobre la oposición binaria de hombre y mujer desde los siguientes campos: como el torturador y la víctima, como un representante del orden y la otra de la subversión; él es un instrumento de la represión y ella, del goce y de la libertad. El Coyote y Roque, el hombre sinnombre, son representantes del discurso hegemónico y parte de una estructura de poder identificada con un sistema político como es la dictadura. Así, sus acciones directa o indirectamente dependen de este gran esquema.

Por otro lado, en "Cambio de armas" es donde mejor se puede observar cómo el cuerpo femenino se ha convertido en el espacio en donde la dictadura textualiza sus mecanismos de represión. Para acentuar la violencia política, el cuerpo de la mujer será el lugar donde se van a operar los atropellos de la dictadura: torturas, violaciones y desapariciones. La identidad perdida de Laura es producto de estas operaciones. Ella, al no tener una identidad está como ausente, no tiene historia. Las dictaduras se caracterizan precisamente porque deforman la Historia y la manipulan. En ese sentido, es significativo cuando el coronel le obliga a recordar quién era ella realmente, la despierta, y le dice: 
- ¿No te das cuenta que esto ya no puede seguir? Basta reaccioná. Se terminó la farra. Mañana a la mañana te van a abrir la puerta y vos vas a poder salir, quedarte, contarlo todo, hacer lo que se te antoje. Total, yo ya voy a estar bien lejos (...). (Valenzuela, 2007, p. 195)

Contar, escribir una versión, hace juego coincidentemente con el primer cuento "Cuarta versión”, es una tarea pendiente en el retorno a la democracia, pues la dictadura ha deformado la historia y debe salir a la luz la verdad. La contaminación de la dictadura es negativa y altera todos los niveles del cuento. Como puede verse, el cuerpo no solo agota su significado en la relación con la identidad femenina sino también se extiende a coger elementos del contexto. La identidad femenina está íntimamente relacionada con el contexto de la dictadura y su liberación es una tarea urgente. Entonces, la acción de empoderamiento es un elemento indesligable de la estructura narrativa que el relato necesita para la construcción de su propia versión de la Historia.

\section{Conclusiones}

$\mathrm{L}$ uisa Valenzuela es una escritora comprometida, ha tenido un papel muy importante capitales dentro de su propia narrativa. En Cambio de armas se puede examinar cómo estas motivaciones se textualizan para resolver en el terreno de la ficción las serias contradicciones que tienen las naciones latinoamericanas en torno a su Historia. Asimismo, se puede observar el rol que desempeña la mujer en este complejo entresijo social y su acción de empoderamiento.

A lo largo de este análisis, se ha examinado cómo la alegoría del cuerpo femenino representa una problematización del complejo proceso político que representó la dictadura en Argentina. Como artefacto cultural y simbólico, este conjunto de relatos propone como soluciones temporales que la escritura de la Historia dependerá en cierta medida del modo en cómo la mujer se apropie de la escritura para redefinir su identidad e intervenga en lo Real para transgredir el discurso que ha oficializado el poder. De esa forma se ha podido demostrar cómo en "Cuarta versión" el sujeto femenino está siempre en una posición transgresora debido a su constante resignificación mediante el acto performativo o de enmascaramiento; al mismo tiempo, quien cumple el rol del narrador debe adjudicarse 
también el proceso de la autoría para concluir un proyecto de narrativización de la Historia en donde la tensión radica en la batalla por fijar el sentido.

Por otro lado, en el relato que da nombre al libro, "Cambio de armas", se concluye que el cuerpo femenino es el espacio en donde la dictadura textualiza sus mecanismos de represión. Así, la relación conyugal entre Laura y Roque, víctima y torturador respectivamente, es un reflejo en la esfera íntima de una situación que ocurre en la esfera pública. En ese sentido, la liberación de Laura solo será posible en la medida que la superación de su amnesia y su proceso de autoconocimiento le permitan apropiarse de su verdadera arma que es el lenguaje.

Finalmente, es relevante notar que el subtexto ideológico de estos relatos implica un acto de empoderamiento. Es decir, una reacción que permita subvertir el orden en donde la mujer ha ocupado tradicionalmente un lugar subordinado y en donde su cuerpo siempre se ha visto torturado, silenciado y reprimido. Estas son, pues, las constantes sobre las cuales la narrativa de Valenzuela ha buscado resolver en la ficción una compleja realidad. 


\section{Referencias}

Bilbija, K. (1996). Maquillaje y escritura en "Ceremonias de rechazo" de Luisa Valenzuela: hacia un cuerpo propio. Inti: Revista de literatura hispánica, 1(43-44), 95-108. Recuperado de http://digitalcommons.providence.edu/inti/vol1/iss $43 / 8$

Callejo, A. (1985). Literatura e irregularidad en Cambio de armas, de Luisa Valenzuela. Revista Iberoamericana, 51(132-133), 575-580. Recuperado de http://revistaiberoamericana. pitt.edu/ojs/index.php/Iberoamericana/issue/view/171/showToc

Hartry, L. (2010). Género, poder y violencia en la obra de Luisa Valenzuela. Boletín Millares Carlo, (29), 333-345. Recuperado de http://mdc.ulpgc.es/cdm/singleitem/ collection/bolmc/id/418/rec/1

Jameson, F. (1989). Documentos de cultura, documentos de barbarie. La narrativa como acto socialmente simbólico (Trad. T. Segovia). Madrid, Espańa: Visor.

Jameson, F. (1994). The Political Unconscious. Narrative as a Socially Symbolic Act (2th ed.). New York, Estados Unidos: Cornell University Press.

Jara, S. (2007). Historia de una relación abyecta en "Cambio de armas" de Luisa Valenzuela. Arrabal, (5), 221-228. Recuperado de http://www.raco.cat/index.php/ Arrabal/article/view/140525

Kantorowicz, E. (2012). Los dos cuerpos del rey. Un estudio de la teología politica medieval. Madrid, España: Akal.

Kaplan, B. (2007). Género y violencia en la narrativa del cono sur 1954-2003. Woodbridge, Reino Unido: Tamesis.

Muñoz, W. (1992). Cambio de armas de Luisa Valenzuela: La aventura de la adquisición de la escritura ginocéntrica. Modern Language Studies, 22(2), pp. 57-71. Recuperado de http: //www.jstor.org/stable/3195018 


\section{RAUF NEME SÁNCHEZ}

Rader, O. (2006). Tumba y poder. El culto politico a los muertos desde Alejandro Magno hasta Lenin. Madrid, España: Siruela.

Saltz, J. (1987). Luisa Valenzuela's Cambio de Armas: Rhetoric of Politics. Confluencia, 3(1), pp. 61-66. Recuperado de http: //www.jstor.org/stable/27921751

Sesana, L. (2003). Procesos de liberación: Cambio de armas: Luisa Valenzuela (Buenos Aires, 1938). Concept, 27. Recuperado de https://concept.journals.villanova.edu/ article/view/150/121

Valenzuela, L. (2007). Cambio de armas. Bogotá, Colombia: Norma.

Vásquez-Medina, O. (2013). Cuerpo, historia y textualidad en Augusto Roa Bastos, Fernando del Paso y Gabriel García Márquez. Madrid, España: Iberoamaericana /Frankfurt, Alemania: Vervuert. 